\title{
Insect Trapping Method Based on Progressive Star Network
}

\author{
http://dx.doi.org/10.3991/ijoe.v11i9.5066 \\ Zhongsheng Qiu ${ }^{1}$, Pengfei Qiu ${ }^{2}$, Qiankun Zhu ${ }^{1}$ and Rong Wang ${ }^{1}$ \\ ${ }^{1}$ Naval Aeronautical and Astronautical University, Yantai Shandong, China \\ ${ }^{2}$ Yantai Academy of Agricultural Sciences, Yantai Shandong, China
}

\begin{abstract}
This paper proposes an insect trapping method based on progressive star network. There are two steps: Firstly, construct the network; secondly, trap insects through the network. The network is constructed as following descriptions: 1 . Confirm the density of light node. 2. Adjust the height of light node versus growth of crops/plants. This paper contributes to relevant studies in that it proposes a new star network, in which the height of light node changes according to the growth of crops or plants, makes full use of biological resources and avoids bad consequences that traditional chemical pesticides bring about. The star network proposed is simple, reliable and successfully avoids redundant data resulted from frequency communication
\end{abstract}

Index Terms-Pests, Star network, Sensor, Wireless.

\section{INTRODUCTION}

Pests are defined as insects that are detrimental to human beings. In other word, pests are insects that generate negative influence on our survival. Whether a kind of insect is harmful or not has much to do with time, location and number of the species. Sometimes phytophagous insects are small in number and low in density, and do not cause damage to crops. Under such circumstances, they cannot be labeled as pests, and there is no necessary to take preventative measures. There are occasions when pests feed their enemies, though in a small population, and enable their enemies to survive in the area, which increases the complexity and stability of the ecological system; they should be treated as beneficial insects [1-3].

Currently, mature biological control methods include: using enemies of insects; using pathogenic microorganisms of insects; using pheromones of insects; using new emerging biological technologies such as RNAi. However, these technologies are lack of innovation. Types of enemies are rare, drug products are limited, supporting technology is not advanced and there also lacks the comprehensive biological control technology and standards prepared for coordinative development between crops and pets and targeting at different areas and various types of agroecological systems [4-6].

The State Intellectual Property Bureau publicized the patent of a progressive star network for insect trapping. The patent number is CN201410459265 and the publication number is CN104255684A. This creation proposes a method using insect-trapping lamp with fixed height. But it fails to consider that the effective distance between the lamp and crops would change versus growth of crops.

The realization of the star network is based on using sense organ of pests. It realizes the progressive trapping of insects under the premises that pests have phototaxis.

\section{BASIC KNOWLEDGE}

(1) Phototaxis pests

Phototaxis pests are born with a special kind of pigment on the retina. This kind of pigment can attract certain wavelength of light, cause reactions and stimulate optic nerves. The nervous system guides locomotive organs and cause wings and feet of insects to move towards the light source. Phototaxis pests are sensitive to $320 \mathrm{~nm}-750 \mathrm{~nm}$ waveband of light. But different types of insects have different sensitivity to the same waveband. Thus, we can make use of the sensitivity of pests to a certain waveband and adjust it to the peak to attract pests to lamps [7-10].

(2) Existing insect-trapping technologies using photoreceptor of pests

It is known that current biological insect-trapping technologies, though mature to some extent, have shortcomings, including the waveband is too wide, the light wave is single rather than diversified, the target is not clear. All these result in that the insect-trapping cannot reach expectation. In addition, existing methods require manual clearance, which also brings about a waste of resources. Therefore, modern ecological agriculture is calling upon a self-organized, progressive way of insecttrapping technology, one that has clear target and wide application. Such technology will realize an environmentally friendly result from "alluring-trappingfeeding" and create an energetic green ecological system [11-14].

\section{REAL PRACTIVE AND RESULTS}

To construct the progressive star network, in each light node there should be distributed LED insect-trapping lamp, lamp driving circuit, 315 wireless transmitter modules, solar panel and microprocessor (on the edge of the network radar sensors should be placed). The equipment should also be applied to the insect-trapping target point. The microprocessor, as the core of the system, controls the time to work under certain calculation. It can automatically calculate at what time to close (or open) insect-trapping lamps and send timely signal to lamps with radar sensors. Once received the 
signal, the radar sensor would check the number of pests and close (or open) the insect-trapping lamps.

Details of how the network functions are explained as below with illustrations:

Firstly, the distribution of light nodes. According to the calculation as mentioned before, at each node is distributed an insect-trapping lamp of height $\mathrm{H}_{\mathrm{x}}$. It has been calculated according to the size of the farmland that the lamps are arranged at every $80 \mathrm{~m}$. The number of lamps is reduced increasingly from the edge to the pond. Finally, all pests are allured to one point above the pond, as shown in Fig.1 (lamps in the same line have the same color of lampshade. Each line of lamp has one particular color).

The lamps are distinguished from their colors. As shown in Fig.4, the local graph of insect-trapping lamps, lamps in the same line have the same color of lampshade. Lamps in different lines have different colors. At each light node, there set up 315 wireless transmitter modules (with the same transmission frequency). Radar sensors are circularly placed in the lamps in the outermost right line. During the insect-trapping process, lamps at all light nodes are opened. Pests will be attracted to the nearest

lamp. After time $\tau$, which is calculated by the microprocessor, the signal is sent to lamps with radar sensors in the first line. Start the radar sensors and check whether pests have increased in numbers. If not, close

lamps in this line. After time $\tau$, microprocessors send the signal again to start the radar. Radar sensors in the second line inspect whether pests have increased in numbers. If not, close the lamps and radar sensors in the second line. This step is repeated until pests are allured to the lamp with the controlling center.

The crop area is a limiting factor. From Table II, it is known that the largest accumulative flying distance of pests is $6500 \mathrm{~m}$. And the distance between two neighboring lamps is $80 \mathrm{~m}$. So, there are 81 lines of insecttrapping lamps. Therefore, if the crop area extends to more than $6500 \mathrm{~m}$, when lamps in the $81 \mathrm{st}$ line are closed, those in the first line can be reopened and pests would not return halfway (this is to make sure that when the crop area is large, we don't need to wait to reopen lamps in the first line until all pests are allured to the target point). Based on that, we can know when to the reopen lamps in the $n$-th line.

$$
\mathbf{n}=\mathrm{N}-80
$$

Where, $\mathrm{N}$ refers to the insect-trapping lamps closed in the N-th line.

Fig. 2 shows the working mechanism of radar sensor. To facilitate power supply, radar sensors are integrated with light nodes. Transparent panel is added below the light source. They form a certain angle with the electromagnetic wave emitted by the radar sensor. When the radar sensor is opened, adjust the emission angle of the electromagnetic wave until it is vertical to the transparent panel. In this way, the single wave will be reflected vertically and form an enclosure area near the light source.
By detecting the pause signal, the microprocessor will figure out whether the number of pests has increased.

Fig. 3 shows the insect-trapping lamps on the water. The equipment has two layers and each of them equipped with a LED lighting device. In between lies an upsidedown cone interlayers. At the top of the cone lies an air hole. The LEDs in the upper layer are lit. After it is confirmed that no pests are increased, the LEDs in the upper layer will be closed. Pests will fall into the pest collecting can when they are physically tired. A floating ring is needed to add below the equipment to stable it and keep it on the water.

This paper mainly discusses insect-trapping for six types of crops, namely paddy, corn, wheat, soy bean, cotton and vegetables. The enemies of these crops are chilo suppressalis, asiatic corn borer, armyworm, soybean pod borer, cotton bollworm, and pieris rapae respectively.

\section{A. The calculation of light node distribution}

1) The calculation of light node height

The height of the light node is defined as the height between the light node and the ground.

TABLE I.

THE HEIGHT OF CROPS

\begin{tabular}{|c|c|}
\hline The term of crop & The height of crop \\
\hline Rice & $h_{1}=0.5 \sim 1.5 \mathrm{~m}$ \\
\hline Corn & $\mathrm{h}_{2}=3 \sim 5 \mathrm{~m}$ \\
\hline Wheat & $h_{3}=0.3 \sim 1.2 \mathrm{~m}$ \\
\hline Soy & $h_{4}=0.3 \sim 0.9 \mathrm{~m}$ \\
\hline Cotton & $h_{5}=1 \sim 2 \mathrm{~m}$ \\
\hline Vegetable & $h_{5}=1 \sim 2 \mathrm{~m}$ \\
\hline
\end{tabular}

The height $\mathrm{H}_{\mathrm{x}}$ of the light node is expressed as the followings:

$$
\begin{aligned}
& \mathrm{H}_{x}=\mathrm{h}_{x}+1.2,(x=1,2,3,4,5) ; \\
& \mathrm{H}_{x}=\mathrm{h}_{x}+0.7,(x=6) ;
\end{aligned}
$$

Where $\mathrm{H}_{x}$ is the height of the light node; $h_{x}$ is the height of the crop.

2) The calculation of light node density

According to their flying habit, flying ability, active time and life cycle, it is calculated that the minimum accumulative flying time of pests is $1 \mathrm{~h}$.

Suppose the daily minimum accumulative flying distances of pests is $\mathrm{L}$,

$$
L_{x}=\mathrm{t} \times V_{x}
$$

Where $\mathrm{t}$ is the minimum accumulative flying time of pests, and $\mathrm{t}=1 \mathrm{~h}$;

TABLE II.

DAILY FLYING DISTANCES OF PESTS

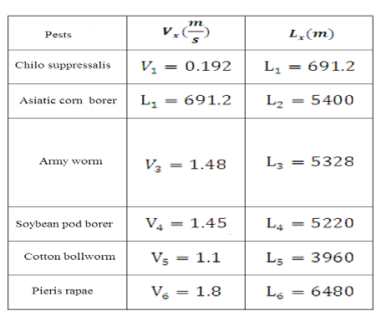


It is calculated that the daily minimum flying distance of pests is more than the effective radius and finishing the daily flight is within the life cycle of pests. Thus, the insect-trapping lamps are distributed at every $80 \mathrm{~m}$.

\section{B. The calculation of insect-trapping progressive star network}

1) The calculation of navigation

This paper is innovative in that it gathers pests in a progressive way and then feed them to fish. The light is turned to navigation. Pests within a certain range are allured to the pond through the progressive navigation of turning on and off lamps. Such method is highly efficient and creates a convenient way to feed the fish. To avoid the condition where pests fly halfway and return, we open all insect-trapping lamps and close them progressively. This part gives a full illustration of the calculation of time to open and close the insect-trapping lamps.

The calculation of density involves effective area of radar sensor, flying distance of pests within effective alluring area and crop area.

Suppose within the effective alluring area, the time of pests flying to the light source is $t_{x}$,

$$
t_{x}=\frac{R}{V_{x}}
$$

Where $\mathrm{R}$ is the effective radius of the circle area to catch pests, and $\mathrm{R}=80 \mathrm{~m}$. $V_{x}$ is the flying speed of pests.

TABLE III.

FLYING SPEED AND LENGTH OF TIME OF PESTS

\begin{tabular}{|c|c|c|}
\hline Pests & $\mathrm{V}_{x}\left(\frac{\mathrm{m}}{\mathrm{s}}\right)$ & $\mathrm{t}_{x}(s)$ \\
\hline Chilits suppresesalis & $\mathrm{V}_{1}=0.192$ & $\mathrm{t}_{1}=416$ \\
\hline Asiatic com borer & $\mathrm{V}_{2}=1.5$ & $\mathrm{t}_{2}=53.3$ \\
\hline Ammy wom & $\mathrm{V}_{3}=1.48$ & $\mathrm{t}_{3}=54.1$ \\
\hline Sostean pod borer & $\mathrm{V}_{4}=1.45$ & $\mathrm{t}_{4}=55.2$ \\
\hline Cotton bollworm & $\mathrm{V}_{5}=1.1$ & $\mathrm{t}_{5}=72.7$ \\
\hline Pieris rapac & $\mathrm{V}_{6}=1.8$ & $\mathrm{t}_{6}=44.4$ \\
\hline
\end{tabular}

Suppose at time $\tau$, the insect-trapping lamp turns on or off.

$$
\tau=\mathrm{t} 1
$$

At 426s, if the radar sensor confirms that no pests are increased, insect-trapping lamps in this area can be turned off.

As shown in Fig.1, the rectangular area is the effective area of crops. Insect-trapping lamps are kept $80 \mathrm{~m}$ with neighboring lamps. Lamps of different lines have different colors of lampshade. The point in the bottom is the target point of insect-trapping.

These lamps divide many areas of the same size

(80m*80m square). Open all lamps and after time $\tau$, the controller at the target point sends the signal to start radar sensors which are used to check whether pests have increased in numbers. If not, close lamps in this line. The process is repeated until all pests are allured to the pond. From Fig. 4 it is known that the daily maximum flying distance of six types of pests is $6500 \mathrm{~m}$. When the crop area extends to more than $6500 \mathrm{~m}$, lamps in the 81 st line can be closed and those in the first line can be reopened to catch pests as many as possible.

How to use the radar sensor to check whether the number of pests is increased is described as below.

The radar sensor used in this paper is an HB100 microwave mobile sensor module. It uses Doppler shift effect to monitor the movement of animals so as to decide a proper time to open or close lamps.

The Doppler shift effect is defined as the relative movement between the emitter and the receptor. The receptor has a different frequency from the emitter which emits information. The phenomenon is known as Doppler effect and the frequency difference is known as the Doppler shift effect. The Doppler shift effect can also be found in the spread of sound. When the sound source has relative movement with the receptor, there occurs the Doppler shift effect $\Delta \mathrm{f}$ between the frequency of sound wave $f^{\prime}$ of the receptor and that of the sound source $f$ :

$$
\Delta \mathbf{f}=\mathrm{f}^{\prime}-\mathbf{f}
$$

When the receptor is moving close to the sound source, the receipting frequency $\mathrm{f}^{\prime}$ is larger than the emitter frequency $\mathbf{f}$ :

$$
\Delta f>0
$$

When the receptor is moving far away from the sound source, the receipting frequency is smaller than the emitter frequency $\mathbf{f}$ :

$$
\Delta \mathrm{f}<0
$$

When the receptor and the sound source are close to or far from each other at speed $\mathrm{V}$, the sound speed $\mathrm{c}$, it can be proved that the Doppler frequency of the receptor is:

$$
\mathrm{f}^{\prime}=\mathrm{f}(\mathrm{c} \pm V 1) /(\mathrm{c} \pm V 2)
$$

The plus sign refers to "close to" and the minus sign refers to 'far from'. When the electromagnetic wave touches the pests and are inflected to the wave source, the sensor will calculate the location of pests according to Doppler shift effect. If no more pests are detected within certain period of time, these lamps can be closed and lamps in the next line can be opened.

To eliminate noises, transparent panel is added below the light source. They form a certain angle with the electromagnetic wave emitted by the radar sensor. When the radar sensor is opened, adjust the emission angle of the electromagnetic wave until it is vertical to the transparent panel. In this way, the single wave will be reflected vertically and form an enclosure area near the light source. By detecting the pause signal, the microprocessor will figure out whether the number of pests has increased. The microwave emitted by the sensor is $10.525 \mathrm{GHz}$, a frequency that is able to detect pests and pose no harm to human beings, as shown in Fig. 2.

Parameters of sensor are shown below: 
TABLE IV.

PARAMETER OF THE RADAR SENSOR

\begin{tabular}{|c|c|c|}
\hline \multirow{4}{*}{ Physical indicator } & Weight & $9 \mathrm{~g}$ \\
\hline & Size & $45 \mathrm{~mm} \times \mathrm{x}: 37 \mathrm{~mm} \times \mathrm{x} \cdot 9.3 \mathrm{~mm}$ \\
\hline & Operating temperature & $-10 \sim+55^{\circ} \mathrm{C}$ \\
\hline & Storage tempecrature & $-30 \cdots+70^{\circ} \mathrm{C}$ \\
\hline \multirow{5}{*}{ Emission indicator } & frequency & $10.525 \mathrm{GHz}$ \\
\hline & power output & $15 \mathrm{dBm} m \cdot \mathrm{EIRP}$ \\
\hline & operating voltage & $+5 \mathrm{v}(+/ / 0.25 \mathrm{v})$ \\
\hline & operating current & $40 \mathrm{~mA}$ (60ma:max) \\
\hline & spurious emission & $<-10 \mathrm{dBm}$ \\
\hline \multirow{2}{*}{ Receiving indicator } & sensitivity & (10dB SNR ) $-90 \mathrm{dBm}$ \\
\hline & noise & $<10 u V$ \\
\hline \multirow{3}{*}{ Antenna } & transmission gain & $10 \mathrm{dBi}$ \\
\hline & $-3 \mathrm{~dB}$ horizontal & $85^{\circ}$ \\
\hline & $-3 \mathrm{~dB}$ vertical vieiw angle & $38^{\circ}$ \\
\hline \multirow{3}{*}{ Pulse mode } & average current $5 \%(\mathrm{DC})$ & 2mA-Typ \\
\hline & pule width (minimum) & 5us \\
\hline & duty cycle(maximum) & $1 \%$ \\
\hline
\end{tabular}

2) The calculation of fish feeding device

The lamps are set above the pond and pests fly around it. A special type of insect-trapping lamp is used to allure it to above the pond. And through special design, pests are hard to fly out, as shown in Fig.3. Pest collecting can is also put above the water and changed on a regular basis.

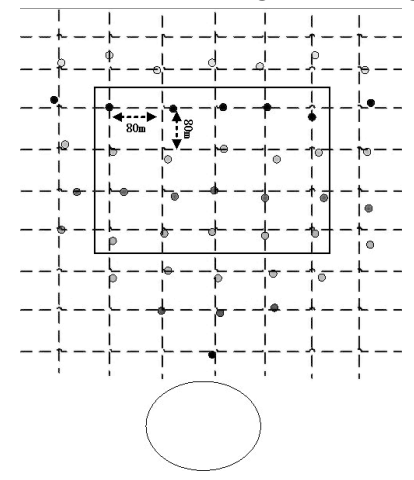

Figure 1. The local distribution of insect-trapping lamps

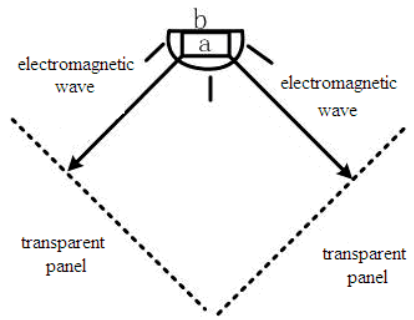

$\mathrm{a}$ is radar sensor, $\mathrm{b}$ is node of LED insect-trapping lamp

Figure 2. The working mechanism of radar sensor

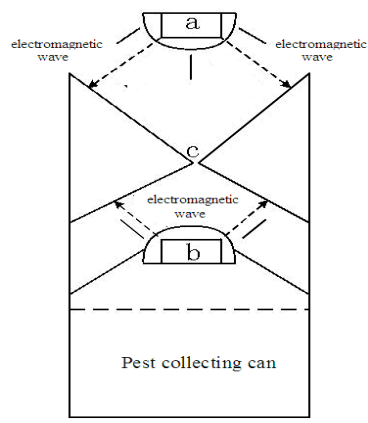

$\mathrm{a}$ is radar sensor, $\mathrm{b}$ is node of LED insect-trapping lamp

Figure 3. The insect-trapping lamps on the water

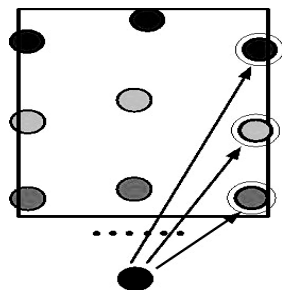

Figure 4. The local graph of insect-trapping lamps

\section{CONCLUSIONS}

In conclusion, the progressive star network is able to allure pests in certain crop area to the pond and feed them to the fish, which makes full use of ecological resources and establishes a new ecological type. More importantly, the star network is simple in communication, reliable and stable.

\section{REFERENCES}

[1] Wu Fuzhen, Guan Zhihe, Ma Shijun, et al. China's Agricultural Encyclopedia: Insects [M]. Beijing: China Agriculture Press, 1990. 343.

[2] Ding Yanqin. Mathematical Ecology of Insects [M]. Beijing: Science Press, 1994.339.

[3] Su Myeong Hong, Zaw Win Min, Chulkyoon Mok. Aqueous degradation of imidacloprid and fenothiocarb using contact glow discharge electrolysis: Degradation behavior and kinetics [J]. Food Science and Biotechnology, 2013, 22(6): 1773-1778. http://dx.doi.org/10.1007/s10068-013-0279-2

[4] Whayne Jeannie. The Incidental Environmentalists: Dale Bumpers, George Templeton, and the Origins of the Rosen Alternative Pest Control Center at the University of Arkansas [J]. AGRICULTURAL HISTORY, 2015, 89 (1): 3-28. http://dx.doi.org/10.3098/ah.2015.089.1.3

[5] Tayade Shailesh, Patel Z P, Singh Susheel, Phapale A D. Effect of weather parameters on pest complex of banana under heavy rainfall zone of South Gujarat [J]. JOURNAL OF AGROMETEOROLOGY. 2014, 16(2): 222-226.

[6] De Oliveira Charles Martins, Dianese Alexei de Campos, Frizzas Marina Regina, Guimaraes Tadeu Graciolli, Campos Gustavo Azevedo. First report of an insect pest on Passiflora tenuifila Killip (Passifloraceae) [J]. PHYTOPARASITICA, 2014, 42(5): 677-680. http://dx.doi.org/10.1007/s12600-014-0408-3

[7] Li Zhao Qun, Zhang Shuai, Luo Jun-Yu, Wang Chun-Yi, Lv Li Min, Dong Shuang Lin, Cui Jin Jie. Ecological Adaption Analysis of the Cotton Aphid (Aphis gossypii) in Different Phenotypes by Transcriptome Comparison [J]. PLOS ONE, 2013, 8(12) http://dx.doi.org/10.1371/journal.pone.0083180

[8] Gemmell Neil J, Jalilzadeh Aidin, Didham Raphael K, Soboleva Tanya, Tompkins Daniel M. The Trojan female technique: a novel, effective and humane approach for pest population control [J]. PROCEEDINGS OF THE ROYAL SOCIETY BBIOLOGICAL SCIENCES, 2013, 280(1773): 20132549. http://dx.doi.org/10.1098/rspb.2013.2549

[9] Vidart Maria Valeria, Mujica Maria Valentina, Calvo Maria Victoria. Relationship between male moths of Cryptoblabes gnidiella (Milliere) (Lepidoptera: Pyralidae) caught in sex pheromone traps and cumulative degree-days in vineyards in southern Uruguay [J]. SpringerPlus, 2013, 2(1): 258. http://dx.doi.org/10.1186/2193-1801-2-258

[10] MacDonald Alexander, Wolf Erick. The political and social barriers for contraception in pest birds: A CASE STUDY OF OVOCONTROL (R) (NICARBAZIN) [J]. JOURNAL OF ZOO AND WILDLIFE MEDICINE, 2013, 44(4): S132-S134. http://dx.doi.org/10.1638/1042-7260-44.4S.S132

[11] Liu Lichun, Gu Guohua, Yang Guxin. The Application of Double Wave Trapping Lamp in Pest Forecast [J]. Entomological Knowledge, 1993, 30(3): 161—166. 
[12] Li Guanbao, Yan Feng, Huang Yu. Xinjiang cotton insect pests integrated control technology popularization case analysis $[\mathrm{J}]$. Rural Science and Educationa, 2014, 11(2): 96-99.

[13] Xu Weimin, Bian Juanshi, Huang Songyu, et al. The Application of Double Wave 4 Lights in the Prediction and Prevention of Helicoverpa Armigera [J]. Jiangsu Agricultural Sciences, 2000, (3): $36-38$.

[14] Ling Xiaoming, Feng Xinjun, Lu Junliang. The Effect of Frequency-Vibrancy Pest-Killing Lamp against Helicoverpa Armigera [J]. Journal of Zhejiang Agricul Tural Sciences, 2000 , (2): $78-80$.

\section{AUTHORS}

Zhongsheng Qiu is with Naval Aeronautical and Astronautical University, Yantai, Shandong, 264001,
China (e-mail: overflyq@yahoo.com).

Pengfei Qiu is with Yantai Academy of Agricultural Sciences, Yantai, Shandong, 264001, China (e-mail: 676510pf@yahoo.com).

Qiankun Zhu is with Naval Aeronautical and Astronautical University, Yantai, Shandong, 264001, China (e-mail: zqk0022@yahoo.com).

Rong Wang is with Naval Aeronautical and Astronautical University, Yantai, Shandong, 264001, China (e-mail: wrrong@yahoo.com).

Submitted 21 September 2015. Published as resubmitted by the authors 20 October 2015. 